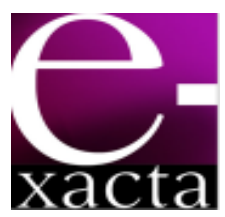

ISSN: 1984-3151

\title{
OTIMIZAÇÃO DO SISTEMA DE CONTROLE DE LARGURA DO ENROLADOR DE MÁQUINAS DE TREFILAÇÃO SUBMERSA
}

\section{OPTIMIZATION OF THE CONTROL SYSTEM WIDTH OF REEL SUBMERGED} WIREDRAWING MACHINES

\author{
João de Oliveira Chaves; Igor Amariz Pires; Arlete Vieira da Silva \\ Centro Universitário de Belo Horizonte, Belo Horizonte, MG \\ joaoochaves@gmail.com; igor amariz@yahoo.com.br; arlete.silva@prof.unibh.br
}

Recebido em: 28/06/2011 - Aprovado em: 07/07/2011 - Disponibilizado em: 24/07/2011

\begin{abstract}
RESUMO: Este estudo aborda a otimização do sistema de controle de largura do enrolador de máquinas de trefilação submersa, utilizando a metodologia FTA, aliada a soluções de automação industrial e à análise gráfica de sinais. O projeto desta solução foi baseado em problemas ocorridos no processo de distribuição do fio trefilado no carretel enrolador, que não ocorre de forma adequada, causando o fenômeno conhecido como mau enrolamento. Para isso foi criado um procedimento para ajuste, realizado a partir de um estudo do algoritmo do CLP, utilizado na supervisão. Neste estudo percebeu-se a ineficiência e a necessidade de modificação deste programa, para validação da eficácia do novo sistema de controle foram desenvolvidos dois programas: o aquisição. exe e o leitura.exe. No desenvolvimento dos programas foi utilizada linguagem gráfica de programação, recurso disponibilizado pelo software LabVIEW 8.0 da National Instruments. O resultado mais relevante demonstrou que houve uma redução de $35 \%$ no número de intervenções no sistema de controle de largura do carretel enrolador, realizadas pela área de manutenção. Esta redução proporcionou maior disponibilidade dos técnicos de manutenção para realizarem outras atividades, aumento da disponibilidade das máquinas para a produção, redução de produto não conforme, devido ao mau enrolamento do fio trefilado. Conclui-se, portanto, que as ferramentas utilizadas mostraram-se confiáveis e eficazes na análise dos dados necessários para a implementação da otimização do sistema de controle de largura, o qual conferiu maior estabilidade e economia ao processo de produção do fio trefilado.

PALAVRAS-CHAVE: Trefilação. FTA, LabView. CLP.
\end{abstract}

ABSTRACT: Nowadays, the FTA methodology is recognized as one of the best tools for the systemic analysis of flaws, being widely utilized for the optimization of complex systems in industries such as aviation, steel, automotive, among others. Therefore, this paper explores the process optimization for spool width control in wet wiredrawing by PLC programming and graphic signal analysis. This study was necessary due to the occurrence of problems related to the inhomogeneous distribution of steel wire filaments on the spool (bad winding). This issue required a greater number of maintenance interventions. In order to implement the optimization of the winding control system, it was necessary to know the components and algorithms. The need to modify, improve code efficiency, and validate the effectiveness of the new system demanded the development of two new softwares: the "Aquisição.exe" and "Leitura.exe". For this development, graphical analysis was acquired with LabVIEW 7.0 (National Instruments ${ }^{\circledR}$ ). Results demonstrated $35 \%$ reduction in the number of interventions related to bad winding. They also showed a reduction on the amount of scrap materials and also raised the machinery productivity, allowing greater availability of the maintenance technicians to perform other more important tasks. In conclusion, the utilized tools have shown to be reliable and effective for the analysis/optimization of the spooling system, resulting greater stability and economy to the wet wiredrawing process.

KEYWORDS: Wiredrawing. FTA. LabVIEW. PLC. 


\section{INTRODUÇÃo}

Atualmente existem muitos fabricantes de produtos, que utilizam o fio trefilado como matéria prima, dentre eles tem-se: cordas para instrumentos musicais, cabos de aço, arame farpado, telas, ferragens para construção civil, aros de bicicletas, cabos de aço para reforço de pneus e mangueiras de alta pressão, entre diversas outras aplicações.

A trefilação de fios para a fabricação de cabos de aço para reforço de pneus apresenta muita complexidade nos processos produtivos e, em conseqüência, também alguns problemas surgem reduzindo a qualidade do produto e causando perdas de produção.

Dentre os problemas que podem surgir no processo está o controle de largura do enrolador das máquinas de trefilação submersa.

Este trabalho aborda o projeto e a implementação de automação industrial, para otimização do sistema de controle de largura do enrolador de máquinas de trefilação submersa, minimizando problemas de enrolamento, reduzindo o número de intervenções de manutenção, buscando a melhoria na produtividade, aumento da disponibilidade de máquinas, redução de produto não conforme. Este trabalho tem como base de implementação a área de trefilação submersa de uma empresa do ramo de arames do Estado de Minas Gerais. A empresa é especializada na fabricação de arames de reforço para pneus radiais de caminhões e utilitários e mangueiras de alta pressão.

Uma das anomalias encontradas frequentemente nas máquinas de trefilação submersa é no sistema de controle de largura do enrolador. Neste processo existe um equipamento que distribui o fio de aço trefilado uniformemente no carretel, entretanto em alguns casos essa distribuição não ocorre conforme previsto, causando o mal enrolamento. Esta não conformidade acarreta em várias intervenções da manutenção, perdas para todo o processo produtivo e, em conseqüência, perdas financeiras para a empresa.
Baseado no exposto questiona-se: como reduzir o mau enrolamento do fio trefilado no carretel, com vista a otimizar o sistema de controle de largura do enrolador das máquinas de trefilação submersa?

Como objetivo principal este estudo se propõe a otimizar o sistema de controle de largura visando reduzir o mau enrolamento do fio trefilado no carretel, reduzindo o número de intervenções da manutenção melhorando assim a performance da área de trefilação submersa da empresa pesquisada.

Como objetivos específicos têm-se: Pesquisar junto ao software de gerenciamento SAP (Systems Applications and Products) os defeitos mais freqüentes nas máquinas de trefilação submersa; Aplicar a ferramenta da qualidade FTA (Fault Tree Analysis) para analisar as falhas detectadas e priorizar as ações corretivas; Aprofundar o estudo do software LabVIEW na parte de armazenamento, consulta e manipulação de dados de forma a gerar informação e conhecimento; Simular o funcionamento do carrinho do vai e vem (carrinho que distribui o fio trefilado no carretel) para auxiliar na solução do problema; Realizar a aquisição dos dados e elaborar um banco de dados para acompanhar a evolução do trabalho.

O presente estudo procura entender melhor o funcionamento do sistema de controle de largura do enrolador, identificando as principais causas que afetam o processo e propor soluções que atenuem, de forma efetiva, as perdas.

\section{TREFILAÇÃo}

Trefilação é um processo em que a matéria prima (fio máquina) é estirada através de uma matriz em forma de um canal convergente (fieira ou trefila) por meio de uma força de tração aplicada do lado de saída da matriz. A Figura 1 mostra a visão geral de uma trefilaria. (NEWBURY; NOTIS, 2004) 


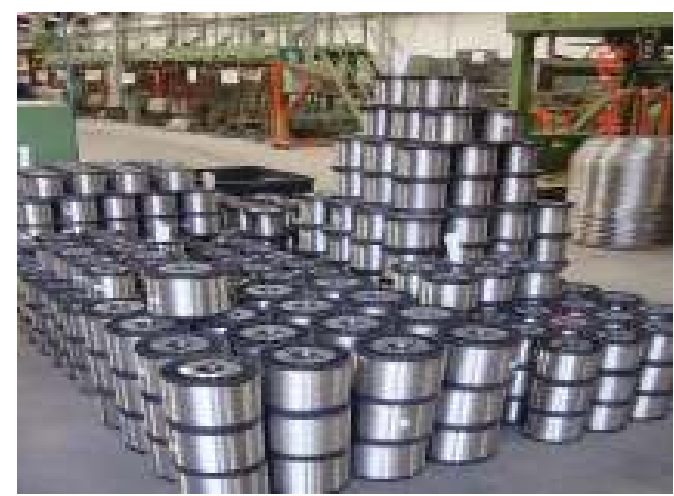

FONTE: Di Martino, 2010.

FIGURA 1: Visão geral de uma trefilaria

Há vários processos de trefilação, porém, aborda-se neste trabalho somente a trefilação a frio que pode ser seca ou úmida (submersa).

No processo de trefilação seca, como geralmente os diâmetros são maiores, utilizam-se as fieiras de vídia. Naturalmente, as deformações muito intensas que se processam durante a trefilação acarretam um aquecimento muito grande dos fios e das fieiras, por esse motivo, tanto as fieiras como os blocos ou cabeças das máquinas, são resfriadas por água, ar ou por ambos (CIMM, 2010).

\section{Fault Tree AnALysis (FTA)}

A FTA é uma ferramenta da qualidade que visa melhorar a confiabilidade de produtos e processos através da análise sistemática de possíveis falhas e suas consequências, orientando na adoção de medidas corretivas ou preventivas (HELMAN; ANDREY, 1995)

O conceito de análise da árvore de falhas surgiu em 1961, desenvolvido por H. A. Watson, do Bell Telephone Laboratories, que queria avaliar o grau de segurança do sistema de controle de lançamentos dos mísseis Minuteman. O processo de construção da árvore tem início com a percepção ou previsão de uma falha, que a seguir é decomposto e detalhado até eventos mais simples. Dessa forma, a análise da árvore de falhas é uma técnica top-down, pois parte de eventos gerais que são desdobrados em eventos mais específicos.

Na Figura 2 é mostrado um exemplo de um diagrama FTA, aplicado a uma falha em um motor de elétrico. O evento inicial, que pode ser uma falha observada ou prevista, é chamado de evento de topo, e está indicado pela seta azul. A partir desse evento são detalhadas outras falhas até chegar em eventos básicos, que constituem o limite de resolução do diagrama. As falhas mostradas em amarelo compõem o limite de resolução deste diagrama.

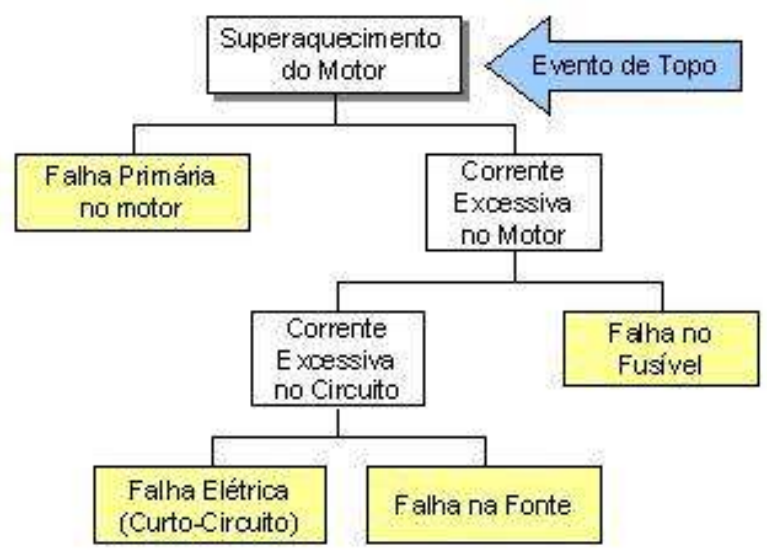

FONTE: Helman; Andrey, 1995.

FIGURA 2: Diagrama FTA

É possível adicionar ao diagrama elementos lógicos, tais como 'e' e 'ou', para melhor caracterizar os relacionamentos entre as falhas. Dessa forma é possível utilizar o diagrama para estimar a probabilidade de uma falha acontecer, a partir de eventos mais específicos.

\section{Principais Finalidades da Ferramenta} FTA

As finalidades da ferramenta FTA são: estabelecer um método padronizado de análise de falhas ou problemas em um equipamento ou processo; compreensão das falhas de um sistema de forma dedutiva; análise da confiabilidade de um produto ou processo; indicação clara e precisa de equipamentos criticos ou condições criticas de operação; compilação 
de informações para treinamento na operação de equipamentos; priorizar as ações corretivas que serão tomadas; análise e projeto de sistemas de segurança ou sistemas alternativos em equipamentos; compilação de informações para planejamento de testes e inspeção; simplificação e otimização de máquinas e equipamentos; compilação de informações para manutenção de sistemas e elaboração de procedimentos de manutenção.

\section{SOFTWARE LABVIEW}

LabVIEW (Laboratory Virtual Instrument Engineering Workbench) é uma linguagem de programação gráfica que utiliza ícones, em vez de linhas de texto, para criar aplicações. Em contraste às linguagens de programação baseadas em texto, em que instruções determinam a execução do programa, o LabVIEW utiliza uma programação baseada em fluxo de dados que determina sua execução. (LabVIEW, 2007)

\section{Metodologia}

Os procedimentos metodológicos foram iniciados com uma revisão bibliográfica a fim de nivelar conhecimento sobre o tema abordado no texto, baseando-se em material já elaborado, constituído principalmente de livros e manuais de equipamentos elétricos. $\mathrm{O}$ trabalho se propõe a vincular a pesquisa teórica à prática, através de um estudo de caso, conforme classificado por Gil (2002), objetivando-se estudar profundamente o sistema de controle de largura das máquinas de trefilação úmida, de maneira que seja alcançada sua compreensão de forma detalhada e que se possa otimizar seu desempenho.

A segunda etapa foi desenvolvida com a finalidade de pesquisar junto ao módulo de manutenção do software de gerenciamento SAP (Systems Applications and Products) defeitos mais frequentes nas máquinas de trefilação submersa e utilizar a ferramenta da qualidade FTA para análise das falhas detectadas e priorizar as ações necessárias para a solução dos problemas.

A terceira etapa consistiu em aprofundar o estudo do software LabVIEW, em relação ao armazenamento, consulta e manipulação de dados de forma a gerar informação e conhecimento da tecnologia utilizada no controle do mau enrolamento e simular com o mesmo software o funcionamento do carrinho do vai-vem (carrinho que distribui o fio trefilado no carretel) para auxiliar na solução do problema.

$\mathrm{Na}$ quarta etapa foram realizados os testes e ajustes no programa do CLP e inversor de frequência, utilizados no controle de largura do enrolador, bem como, a aquisição dos dados com software LabVIEW e SAP com a finalidade de elaborar um banco de dados para acompanhar a evolução do trabalho.

\section{CARACTERIZAÇÃo dA ÁREA DE ESTUDO}

Este trabalho teve como base de implementação a área de trefilação submersa de uma empresa situada no Estado de Minas Gerais. A empresa é especializada na fabricação de cordoalhas de aço, empregados no reforço de pneus radiais e hose reinforcement wire, utilizados no reforço de mangueiras de alta pressão.

A área de trefilação submersa da empresa é composta por 350 máquinas, todas desenvolvidas com tecnologia de ponta. Porém algumas máquinas mais antigas apresentam muitos problemas no sistema de controle de largura do enrolador. Neste processo, existe um equipamento chamado "vai e vem" (Figura 3), o qual distribui, uniformemente no carretel, o fio de aço trefilado. Entretanto, em alguns casos, essa distribuição não ocorre de forma adequada, causando o fenômeno conhecido como mau enrolamento. 


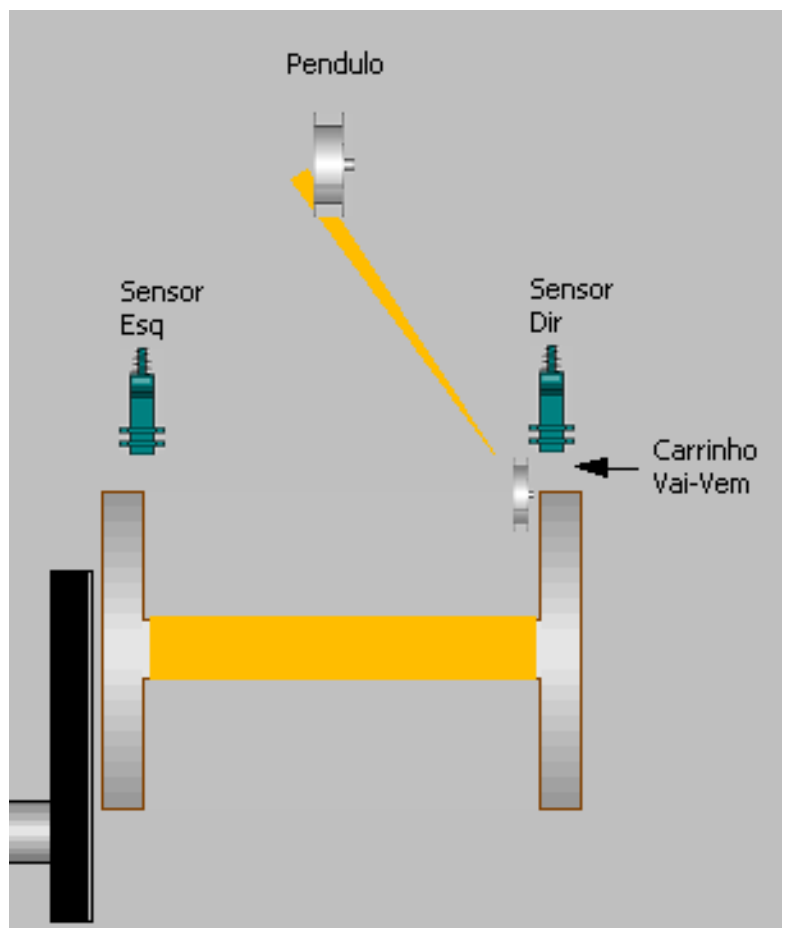

FIGURA 3: Conjunto do vai e vem

Este mau enrolamento (figuras 4 e 5) causa perdas de produção, retrabalho e muitas intervenções da manutenção, além de impactar as fases seguintes do processo.

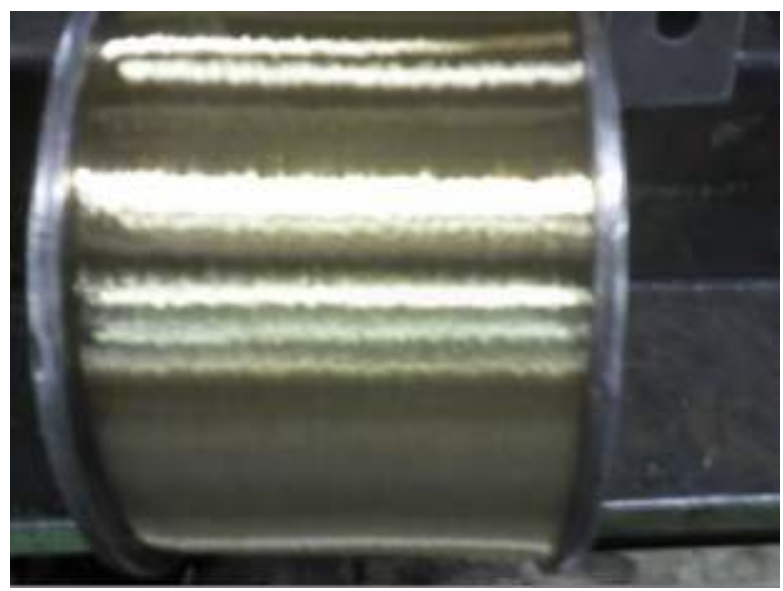

FIGURA 4: Mau enrolamento falta de fio trefilado no lado esquerdo do carretel

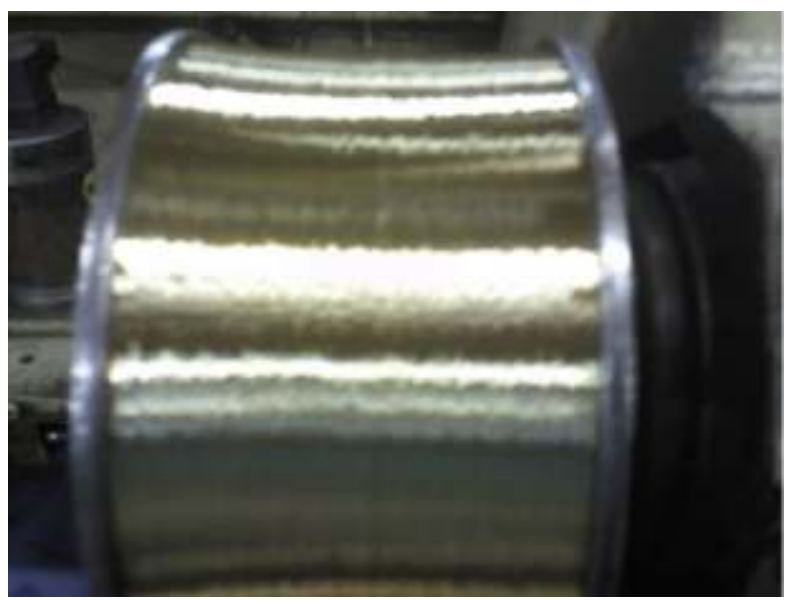

FIGURA 5: Mau enrolamento excesso de fio trefilado no lado esquerdo do carretel

\section{Resultados e Discussão}

Devido ao elevado número de intervenções foi realizada uma pesquisa junto ao módulo de manutenção do software de gerenciamento SAP em que foram verificados os defeitos mais frequentes das máquinas de trefilação submersa no primeiro semestre de 2010 (Tabela 1).

TABELA 1: Defeitos mais frequentes no primeiro semestre de 2010

\begin{tabular}{|c|c|c|}
\hline Componente & $\Delta \nabla$ & Total geral \\
\hline SENSORES DO VAI E VEM & & 145 \\
\hline REGULAGEM DE SENSORES & & 115 \\
\hline FIAÇÃO DO VAI E VEM & & 27 \\
\hline SENSOR DIREITO & & 23 \\
\hline MOTOR DO VAI E VEM (BERGER) & & 20 \\
\hline SENSORES DO PENDULO & & 17 \\
\hline SENSOR ESQUERDO & & 14 \\
\hline Total geral & & 361 \\
\hline
\end{tabular}

O resultado da pesquisa está representado no gráfico de Pareto (Figura 6), onde se pode constatar que um dos defeitos mais frequentes nas máquinas de trefilação submersa foi observado nos sensores do vai e vem, o qual está relacionado diretamente com o sistema de controle de largura do enrolador. 


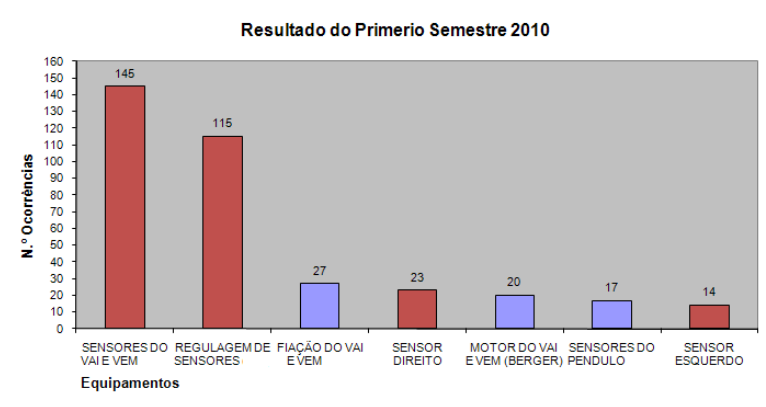

FIGURA 6: Gráfico dos defeitos mais frequentes no primeiro semestre de 2010

Também foi possivel observar no gráfico que todas as ocorrências relacionadas aos sensores do vai e vem estão destacadas em vermelho, totalizando 297 ocorrências, sendo estas distribuídas nos sensores do vai e vem com 145 ocorrências; regulagem de sensores com 115 ocorrências; sensor direito com 23 ocorrências e sensor esquerdo com 14 ocorrências.

\subsection{APLICAÇÃo dA FERRAMENTA FTA}

Após a constatação de que o defeito mais frequente no sistema de controle de largura do enrolador estava relacionado aos sensores do vai e vem que somavam um total de 297 ocorrências, foi montado um grupo de estudo com os profissionais envolvidos na área, criando um diagrama FTA (Figura 7) em que, através da árvore de falhas, possibilitou realizar a análise sistemática das possíveis falhas e suas consequências, orientando na adoção de medidas corretivas e preventivas.

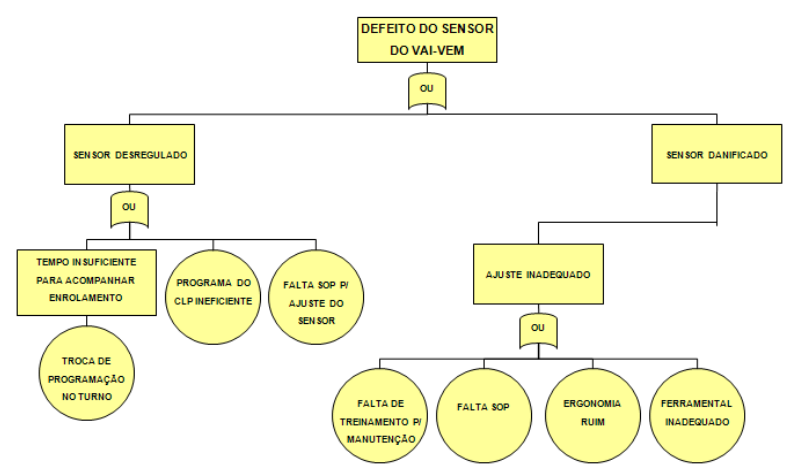

FIGURA 7: Diagrama FTA defeito sensor do vai e vem
Durante a discussão definiu-se os principais tópicos a serem abordados no Plano de Ações: Padronizar ferramenta para ajuste dos sensores do vai e vem; Elaborar SOP (Standard Operating Procedure), procedimento padrão operacional para ajuste do sensores, bem como, treinar os técnicos envolvidos na operação; Concentrar a troca de programação no turno fixo; Verificar programa do CLP e otimizar controle de largura do vai e vem.

Com isso percebeu-se a necessidade do desenvolvimento e a padronização de uma ferramenta (Figura 8) que consistia em um cachimbo $24 \mathrm{~mm}$ soldado em um tubo metálico com uma empunhadura para facilitar o manuseio.

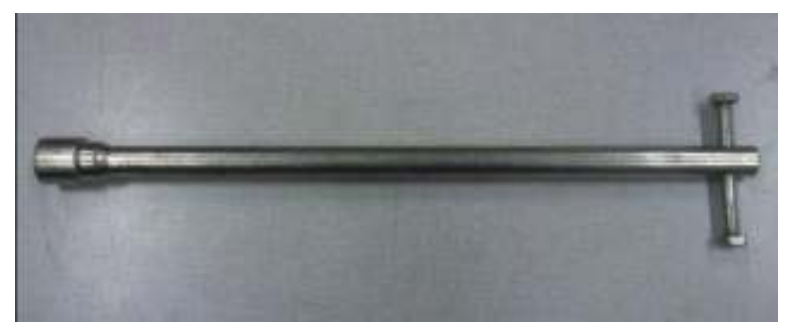

FIGURA 8: Chave para ajuste dos sensores do vai e vem

Esta ferramenta permitiu realizar um ajuste mais rápido e preciso dos sensores do vai e vem, agilizando, assim, a troca de programação da produção e também facilitar a operação por parte dos técnicos, atendendo ao quesito ergonomia.

\subsection{ELABORAÇÃo DO SOP}

Uma vez que não havia um procedimento padrão no momento da troca de programação da produção, onde era necessário o ajuste dos sensores do vai e vem, observou-se que isso acarretava muitos problemas de mau enrolamento e, em consequência, muitas ocorrências para a manutenção. Com o intuito de minimizar este problema, orientar e capacitar os técnicos envolvidos nesta operação foi elaborado um SOP que é composto pelo layout do sistema enrolador (Figura 9), que identifica todos os componentes do 
sistema e dos passos para fazer o ajuste dos sensores do vai e vem.

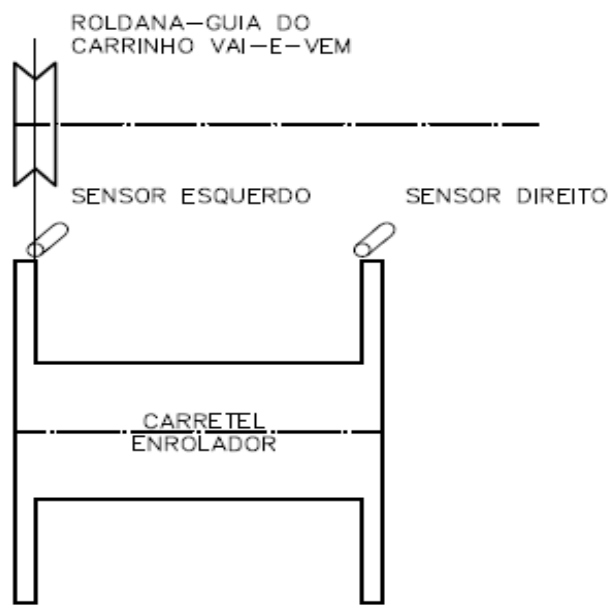

FIGURA 9: Layout do sistema enrolador

Procedimento para ajuste do sensor do lado esquerdo: Posicionar a roldana guia do carrinho do vai e vem alinhada com a aba esquerda do carretel enrolador; Movimentar o sensor para a esquerda até que o mesmo seja desabilitado; Movimentar o sensor para a direita até o primeiro momento, que o sensor seja habilitado; Continuar movimentando o sensor para a direita, afastando-o da aba do carretel por aproximadamente $3 \mathrm{~mm}$.

Procedimento para ajuste do sensor do lado direito: Posicionar a roldana guia do carrinho do vai e vem alinhada com a aba direita do carretel enrolador; Movimentar o sensor para a direita até que o mesmo seja desabilitado; Movimentar o sensor para a esquerda até o primeiro momento que o sensor seja habilitado; Continuar movimentando o sensor para a esquerda afastando-o da aba do carretel por aproximadamente $3 \mathrm{~mm}$.

Após o ajuste dos sensores conforme procedimentos descritos, o técnico deve ligar a máquina e acompanhar o funcionamento. Caso seja necessário, os sensores podem ser ajustados para a direita ou esquerda em até $1 \mathrm{~mm}$, a fim de se obter um funcionamento adequado.

\subsection{ANÁLISE DO FUNCIONAMENTO DO SISTEMA DE CONTROLE DE LARGURA}

No sistema do enrolador há um programa no CLP que detecta todos os sinais dos sensores digitais e analógicos, que faz todo o controle da velocidade do motor enrolador. À medida que o carretel vai enchendo deve-se diminuir a velocidade. O programa do CLP também é responsável pelo sistema de controle de largura do carretel, que determina o momento em que o carrinho do vai e vem deve inverter o sentido de rotação para evitar o mau enrolamento, próximo a aba do carretel.

Nesse estudo de caso foi proposta a otimização do programa do CLP com o intuito de tornar mais eficaz a lógica do controle de largura do enrolamento. A partir do exposto montou-se a seguinte estratégia, que será detalhada nos itens subsequentes: Fazer aquisição de dados com o programa Aquisição.exe LabView, utilizando o programa original do CLP e analisar com o programa Leitura.exe a performance do sistema de controle e largura; Modificar o programa do controle de largura no CLP (implementar otimização); Fazer aquisição de dados com o programa modificado; Fazer comparações dos dois tipos de controle em questão; Apresentação dos resultados.

\subsection{MODIFICAÇÃO DO PROGRAMA DO CONTROLE DE LARGURA NO CLP}

Foram desenvolvidos vários testes e análises no sistema de controle de largura (in loco), o que permite constatar que nem sempre a variação do sinal do pêndulo ao acionar os sensores representava a real situação do enrolamento. Isto ocorre devido a uma série de variáveis, entre elas: deslizamento do fio trefilado na polia de tração, carretel empenado e folga no eixo enrolador. Tais variáveis causavam variações indesejáveis no sinal do pêndulo, o que prejudicava a detecção do mau enrolamento. Outro ponto relevante era a rápida variação dos tempos de correção 
(esquerdo e direito) a cada informação incorreta do pêndulo, contribuindo na formação do mau enrolamento.

Observou-se a necessidade de modificar o programa do CLP. Foi desenvolvido um algoritmo que armazena três aquisições sequenciais do sinal do pêndulo e calcula a média com o objetivo de averiguar a tendência do mau enrolamento. Quando necessário, uma correção automática é feita variando-se o tempo em um valor fixo de $4 \mathrm{~ms}$.

\subsection{COMPARAÇÃO DOS DOIS TIPOS DE CONTROLE}

\section{(ORIGINAL E MODIFICADO)}

Foi feita a comparação das aquisições obtidas dos sinais dos tempos de correção direito (cor laranja) e esquerdo (cor lilás) dos dois produtos em questão, indicado pelas figuras 10 e 11. Pode-se observar com mais clareza a diferença do comportamento do sistema de controle de largura utilizando-se o programa original e o programa modificado. Verificase uma maior estabilidade dos tempos de correção com o programa modificado, por conseguinte, gerando um significativo ganho de performance no controle do enrolamento.
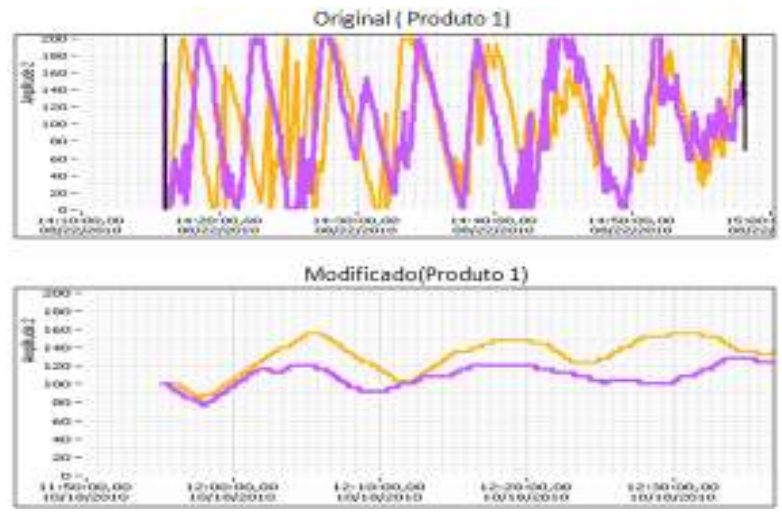

FIGURA 10: Comparação do comportamento do tempo de correção utilizando o programa original e modificado (produto 1)
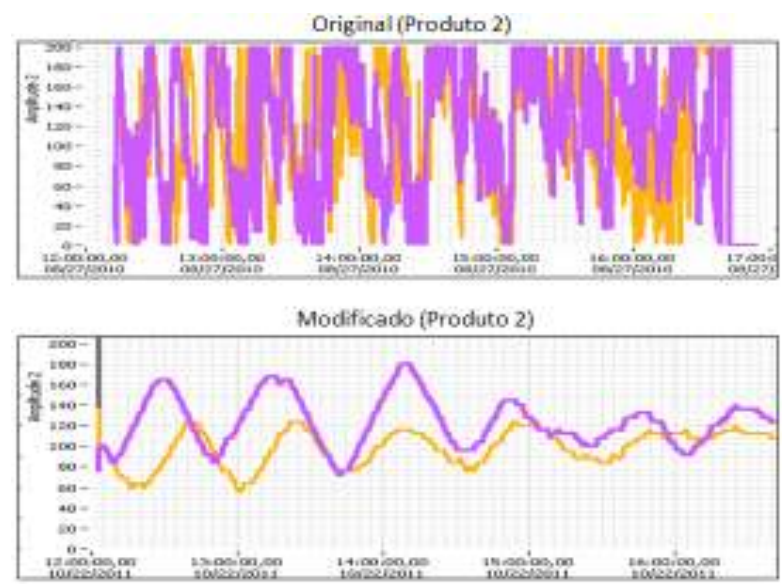

FIGURA 11: Comparação do comportamento do tempo de correção utilizando o programa original e modificado (produto 2)

Consideram-se ainda, para efeito de comparação, os histogramas dos sinais do pêndulo (figuras 13 e 14). Nota-se uma maior estabilidade do sinal do pêndulo através do programa modificado. Neste caso, o valor se manteve estável entre -420 e -430 , em cerca de $58 \%$ das aquisições, significando menos incidência de mau enrolamento próximo às abas do carretel. Já com o programa original a variação foi maior se mantendo entre -420 e -430 , em $45 \%$ das aquisições e, o restante das aquisições, variou de -400 a -450 , portanto como visto anteriormente, a maior variação do pêndulo é indicio de mau enrolamento.

Ao iniciar o processo de trefilação (start da máquina), com o carretel enrolador vazio, inicia-se também o controle de largura do enrolamento, que consiste em analisar a variação do valor analógico do pêndulo no instante em que o carrinho do vai e vem aciona os sensores direito ou esquerdo que estão posicionados próximo às abas do carretel (Figura 12) e a partir dessa informação o algoritmo (programa do CLP) incrementa ou decrementa o tempo correção, que é de fundamental importância na qualidade do enrolamento, este tempo de correção inicia-se com $0 \mathrm{~ms}$ e pode chegar no máximo a $200 \mathrm{~ms}$. Através da variação do tempo de correção o programa do CLP faz o controle de largura tentando manter um 
enrolamento mais uniforme, até que o carretel complete a metragem estabelecida pelo processo e, então, desliga a máquina.

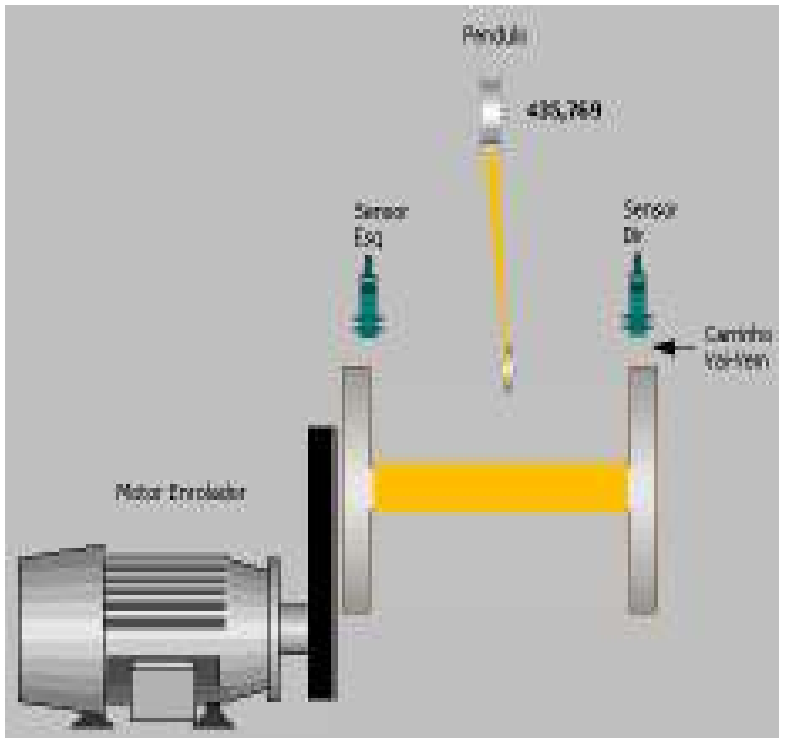

FIGURA 12: Simulação do sistema enrolador

Verificando os histogramas das figuras 13 e 14, podese comprovar a ineficiência do sistema de controle de largura do enrolador pelo programa original do CLP. Observou-se que, nos dois produtos, o tempo de correção esquerdo permaneceu em zero milissegundo na maior parte das aquisições, provocando a falta de fio trefilado (mau enrolamento no lado esquerdo). Verificou-se, também, que o tempo de correção atingiu o valor máximo 200 ms, em várias vezes, o que representa o excesso de fio trefilado. Deste modo, conclui-se que houve um mau enrolamento intermitente durante o enchimento do carretel onde, ora observou-se a falta de fio trefilado, ora o excesso próximo a aba esquerda do carretel.

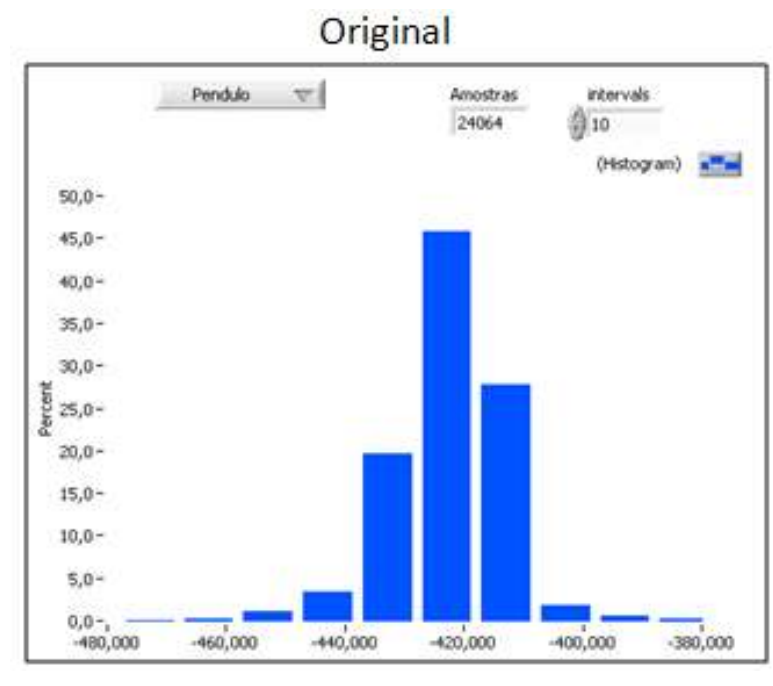

FIGURA 13: Histograma Original

Modificado

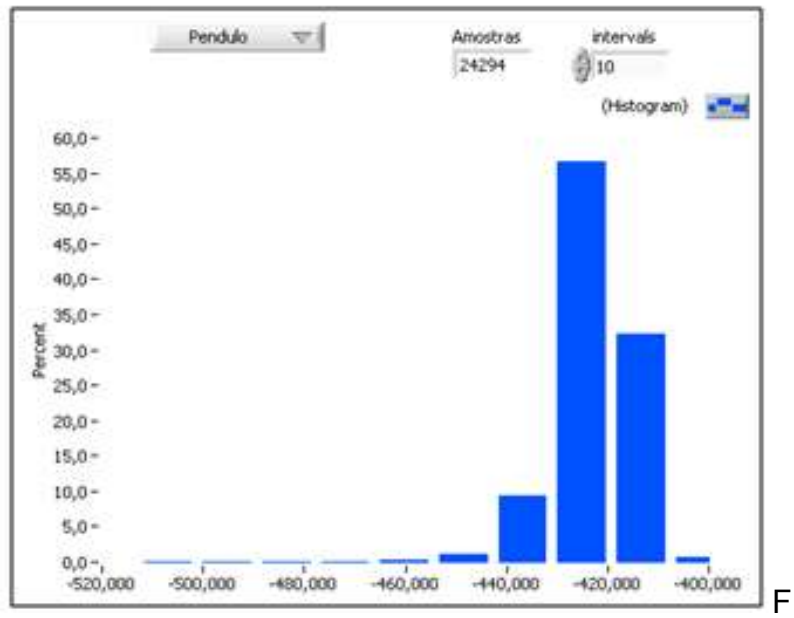

FIGURA 14: Comparação dos histogramas do sinal do pendulo com o programa original e modificado

Após a implementação da otimização do sistema de controle de largura do carretel enrolador, foi realizada uma nova pesquisa junto ao módulo de manutenção do software de gerenciamento SAP. O resultado pode ser visualizado através da Tabela 2. 
TABELA 2: Defeitos mais frequentes no segundo semestre de 2010

\begin{tabular}{|c|c|}
\hline Componente & \begin{tabular}{|l|l|}
$\nabla$ & Total geral \\
\end{tabular} \\
\hline SENSORES DO VAI E VEM & \begin{tabular}{l|r} 
& 95
\end{tabular} \\
\hline REGULAGEM DE SENSORES & 62 \\
\hline FIAÇÃO DO VAI E VEM & 30 \\
\hline MOTOR DO VAI E VEM (BERGER) & 18 \\
\hline SENSORES DO PENDULO & 12 \\
\hline SENSOR DIREITO & 11 \\
\hline SENSOR ESQUERDO & 6 \\
\hline Total geral & 234 \\
\hline
\end{tabular}

Observando-se os resultados relatados anteriormente, verifica-se uma queda no índice de defeitos mais frequentes nas máquinas de trefilação submersa durante o segundo semestre de 2010. Este foi o período de implementação da otimização do sistema de controle de largura. Verifica-se, também, que o número de ocorrências caiu de 361 para 234 o que representa uma redução de $35 \%$.

Este resultado representa um ganho considerável em todo o processo produtivo do fio trefilado e impactando também na fase seguinte do processo. Entre estes ganhos podem-se destacar: melhora no índice de disponibilidade das máquinas de trefilação, melhora de produtividade, melhora da qualidade do enrolamento do fio trefilado, redução do número de intervenções da manutenção, maior disponibilidade dos técnicos de manutenção para realizarem outras atividades e redução de produto não conforme (mau enrolamento).

\section{ConClusão e Considerações FinaIS}

A metodologia FTA utilizada no estudo de eliminação de falhas no sistema enrolador aliada às soluções de automação industrial e à análise de sinais demonstraram ser ferramentas importantes na otimização do sistema de controle de largura do carretel enrolador. Os resultados obtidos através das experiências práticas aplicadas atenderam ao objetivo principal, que era a redução do número de intervenções da manutenção, além de outros objetivos, que agregaram valor ao produto.

Conforme estabeleciam os objetivos propostos, foram alcançados, nesta pesquisa, os seguintes resultados: Redução de $35 \%$ no número de intervenções da manutenção no sistema enrolador das máquinas de trefilação submersa, proporcionando maior disponibilidade para os técnicos de manutenção realizarem outras atividades; Aumento da estabilidade do sistema de controle de largura e economia no processo de produção do fio trefilado; Aumento da disponibilidade das máquinas para a produção; Redução de produto não conforme devido ao mau enrolamento; Aprofundamento do manuseio do software de gerenciamento SAP pela equipe, principalmente no módulo de manutenção, onde podese consultar todos os dados estatísticos relacionados à manutenção de toda planta; Aprofundamento do conhecimento da equipe na linguagem de programação gráfica LabView, especificamente em simulação, aquisição e análise de dados utilizando OPC como forma de comunicação com o CLP.

O que em muitas vezes pode parecer teoricamente simples é algo cercado de considerável complexidade do ponto de vista técnico. É relevante ressaltar a importância desse estudo não somente para a elaboração deste artigo, mas também para possíveis implementações futuras dentro da organização estudada, obedecendo alguns critérios decisivos que definem a segurança e a confiabilidade de suas instalações. Com a finalidade de se atender a tais critérios a busca de conhecimento torna-se uma atividade cotidiana de profissionais, da mesma forma, quando se almeja desenvolver algum tipo de trabalho ligado a este contexto.

A redução do número de intervenções da manutenção no sistema de controle de largura mostrou-se significativa. Porém sugere-se a troca do motor Berger, utilizado no carrinho do vai e vem por um 
motor de indução trifásico controlado por inversor de

sistema.

frequência, o que proporcionaria maior eficiência ao

\section{REFERÊNCIAS}

ASSOCIAÇÃO BRASILEIRA DE NORMAS TÉCNICAS. NBR IEC 60947-2/95: Dispositivos de Manobra e Comando de Baixa Tensão. Rio de Janeiro, 1998. 80p.

BERGER, Lahr. Berger Lahr Mechatronic Basic Products $8 / 2004$. Technical data. Manual de motores Síncronos. Versão de 2004.

BRAGA, N.C. Sensores para todas as aplicações. Saber Eletrônica, São Paulo, n.356, p. 20-22, set. 2002

CAPELLI, A. Sensores. Mecatrônica Atual, São Paulo, n. 4, p. 20-24, jun./jul. 2002

CIMM - Centro de Informação Metal Mecânica. Conformação Mecânica: Trefilação. Disponível em: <http://www.cimm.com.br/portal/noticia /material_didatico/6462>. Acesso em: 10 out. 2010.

CONTROLADORES Programáveis, Disponível em: $<$ www.eletricazine.hpg.ig.com.br/apostilas/eletronica/c ontr_programaveis.zip> Acesso em: 1 nov. 2010

DI MARTINO. DI Martino Indústria Metalúrgica. Disponível em:

<http://www.dimartino.com.br/portanimado/> Acesso em: 1 out. 2010.

EJM Engenharia: Conversores de Frequência e Soft Starters; Disponível em: <http://www.ejm.com.br/Downloads/Inversores.pdf> Acesso em: 5 out.2010.

GIL, Antônio C. Como elaborar projetos de pesquisa. São Paulo, Atlas, 2002.

HELMAN,Horacio;ANDREY,Paulo R.P. TQC: Análise de falhas(aplicação dos métodos FMEA e FTA) . Belo Horizonte, MG: Fund. Cristiano Ottoni, UFMG,1995.

Manual Básico LabVIEW: LabVIEW TM Basics I: Course Manual. Versão 2007 Disponível em $<$ https://lumen.ni.com/nicif/us/trainingbrochure/content. xhtml> acesso em: 4 set. 2010.
MARQUES, L./ SILVA, B. R./ EWALD, B. H./ TEIXEIRA, T. Princípio dos Motores Elétricos. 2009. 08 f. Projeto de Pesquisa - Fundação Escola Técnica Liberato Salzano Vieira da Cunha, Novo Hamburgo, 2009.

MOELLER. PS4: Controlador Lógico Programável Compacto. Disponível em: <http://www.moeller.com.br/produtos/ps4.htm> Acesso em: 20 dez. 2010.

NEWBURY, Brian D.; NOTIS, Michael R.. The History and Evolution of Wiredrawing Techniques. Jomjournal Of The Minerals, Metals And Materials Society: Archaeotechnology, Boston, v. 56, n. 2, p.3337, fev. 2004. Disponível em <http://www.springerlink.com/content/4324q882232h3 275/>. Acesso em: 10 out. 2010.

SIEMENS: Simovert Masterdrives MC; Disponível em:

<http://www.siemens.com.br/templates/produto.aspx?c hannel=250\&produto=14330 $>$ Acesso em: 7 out. 2010 .

TRIVIÑOS, Augusto N. S. Introdução à pesquisa. São Paulo: Atlas, 1987.

UERJ. Curso de Controladores Lógicos Programáveis. Rio de Janeiro, 2000

WEG Motors. E-technical Catalog. Disponível em: $<$ http://www.weglibrary.com/pdf>. Acesso em 05 set 2010.

WEG. CFW 08 Inverter Catalog. Disponível em: $<$ http://www.weglibrary.com/

library_public/index.asp? ID=6\#>. Acesso em: 5 set. 2010. 